\section{Tracheal botryomycosis in a patient with tracheopathia osteochondroplastica}

\author{
Jin-Yuan Shih, Po-Ren Hsueh, \\ Yih-Leong Chang, Li-Na Lee, \\ Yee-Chun Chen, Ming-Fong Chen, \\ Kwen-Tay Luh
}

\begin{abstract}
Tracheal botryomycosis has never been reported in the literature. A 68 year old man presented with progressive dyspnoea and an exophytic mass below the vocal cords was found by bronchoscopy. Pathological study of the mass showed tracheal botryomycosis probably associated with Peptostreptococcus spp infection. The patient was given intravenous penicillin for six weeks. Tracheal obstruction necessitated tracheal resection and the surgical specimen revealed typical findings of tracheopathia osteochondroplastica without residual lesions of botryomycosis. (Thorax 1998;53:73-75)
\end{abstract}

Keywords: botryomycosis, tracheopathia osteochondroplastica, airway obstruction.

Botryomycosis is an uncommon chronic suppurative bacterial infection. Both cutaneous and visceral forms have been described. ${ }^{1-5}$ Visceral botryomycosis is less common and affected organs have included the liver, ${ }^{2}$ kidney, brain, ${ }^{3}$ prostate gland, orbit, tongue, ear, bowels and lung. ${ }^{4}$ However, botryomycosis of the trachea has never been reported.

Tracheopathia osteochondroplastica is an unusual disorder in which nodules of metaplastic bone and cartilage develop in the submucosa of the trachea. ${ }^{6-9}$ This condition is usually asymptomatic and has a benign course. ${ }^{67}$ To date more than 300 cases of tracheopathia osteochondroplastica have been reported, but concomitant bacterial infection of the trachea has not been described in detail.

The case history of a patient with airway obstruction caused by tracheal botryomycosis and tracheopathia osteochondroplastica is presented.

\section{Case report}

A 68 year old man presented with progressive dyspnoea for six months and syncope which had developed just before arrival at the emergency department. He had smoked one pack of cigarettes a day for 40 years. He had suffered diabetes mellitus and hypertension, both under regular medical control, for more than 10 years and had had a stroke about 10 years previously. Progressive exertional dyspnoea, which could be relieved by rest, had bothered him for several months. For the previous two weeks he had experienced episodic dyspnoea with loud breathing sounds, even at rest.

Sudden onset of severe dyspnoea with cold sweating, nausea, dizziness and syncope had occurred before arrival at the emergency department. On examination he was alert, afebrile, slightly tachypnoeic, with diffuse wheezes during the inspiratory and expiratory phase. The chest radiograph was negative. The leucocyte count was $9.2 \times 10^{9} / 1$ (60\% neutrophils, 32\% lymphocytes) and the haemoglobin was $12.1 \mathrm{~g} / \mathrm{dl}$. Tachypnoea and cyanosis followed by respiratory arrest occurred later in the emergency room. After cardiopulmonary resuscitation he was in a comatose state and was admitted to the critical care unit. Extubation was performed on the third day. A foul smell of exhaled air, blood-tinged sputum, episodic dyspnoea, orthopnoea, and stridor were noted. Bronchoscopic examination revealed a whitish exophytic mass with an irregular surface which almost completely occluded the tracheal lumen below the vocal cords (fig 1). The bronchoscope was not advanced further. Tracheostomy was performed immediately. A computed tomographic (CT) scan of the neck revealed an ill-defined mass of soft tissue density in the upper trachea which was excised using laryngomicroscopy. Profuse foul pus drained from the tracheostoma which, when cultured, yielded a heavy growth of Peptostreptococcus species. A second bronchoscopic examination showed that there were multiple whitish ulcerations and nodules on the tracheal wall from the subglottic area to the tracheostoma; the trachea below the stoma, both main bronchi, and the lobar and segmental bronchi were spared. Pathological examination

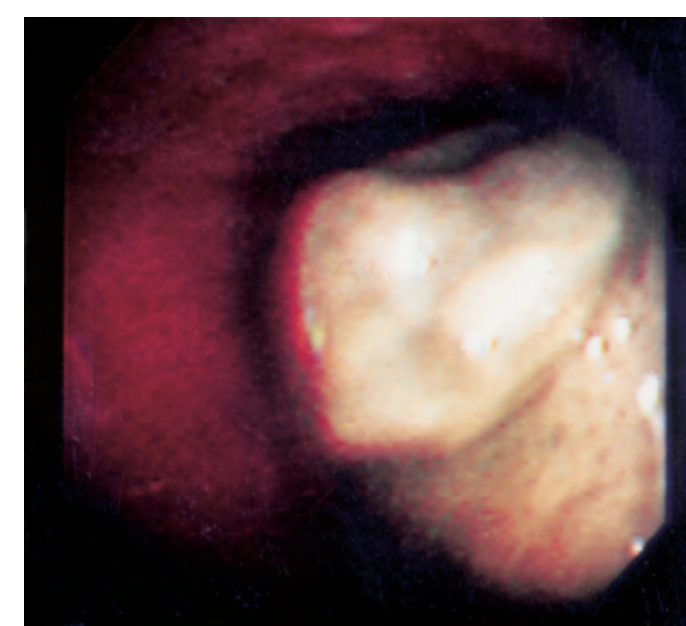

Figure 1 Bronchoscopic examination showing a whitish exophytic mass with irregular surface almost completely occluding the tracheal lumen at the subglottic area. 
Figure 2 Gram stain of the sulphur granule-like lesion showing clusters of Gram-positive cocci surrounded by radially arranged material.

Original magnification $\times 330$.

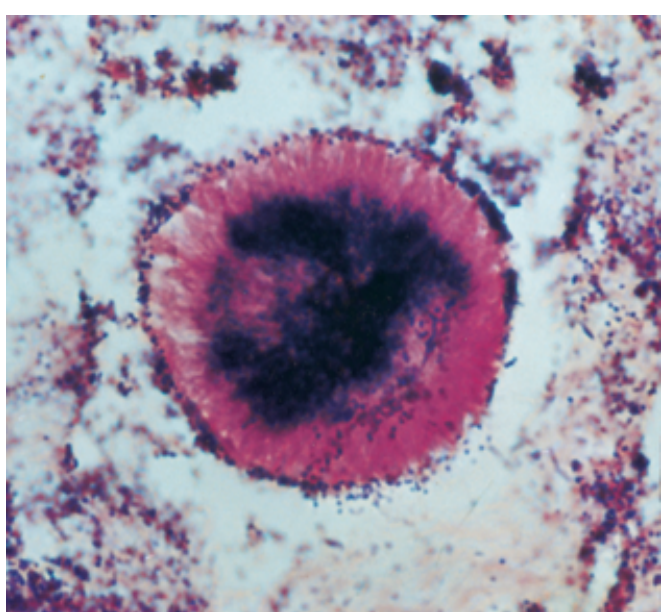

of the excised mass revealed sulphur granulelike material surrounded by a necrotic substance and inflammatory cells on haematoxylin and eosin staining. A fragment of cartilage accompanying the granules was found. Gram staining revealed many clusters of Grampositive cocci scattered within and outside the eosinophilic hyaline material (fig 2). Stains for fungi (periodic acid-Schiff and Gomori's methenamine silver stains) and acid-fast bacilli were negative.

The diagnosis of tracheal botryomycosis was made and treatment with intravenous penicillin was commenced. Anti-HIV antibody was negative and serum levels of immunoglobulin subsets were normal. Lymphocyte subsets determined by flow cytometry demonstrated $74 \%$ CD3+ (normal 74.2 (2.5)\%), 26.4\% CD4+ (absolute CD 4 count $491 / \mathrm{mm}^{3}$ ), $47 \%$ $\mathrm{CD} 8+, \mathrm{CD} 4 / \mathrm{CD} 8$ ratio 0.56 (normal 1.48 $(0.05)$ ), and $9.5 \%$ CD19+ (normal 11.3 $(2.4) \%)$.

After treatment with penicillin for four weeks bronchoscopic examination showed an improvement in tracheal inflammation and diameter but some nodules with a smooth surface were still present on the tracheal wall. Bron-

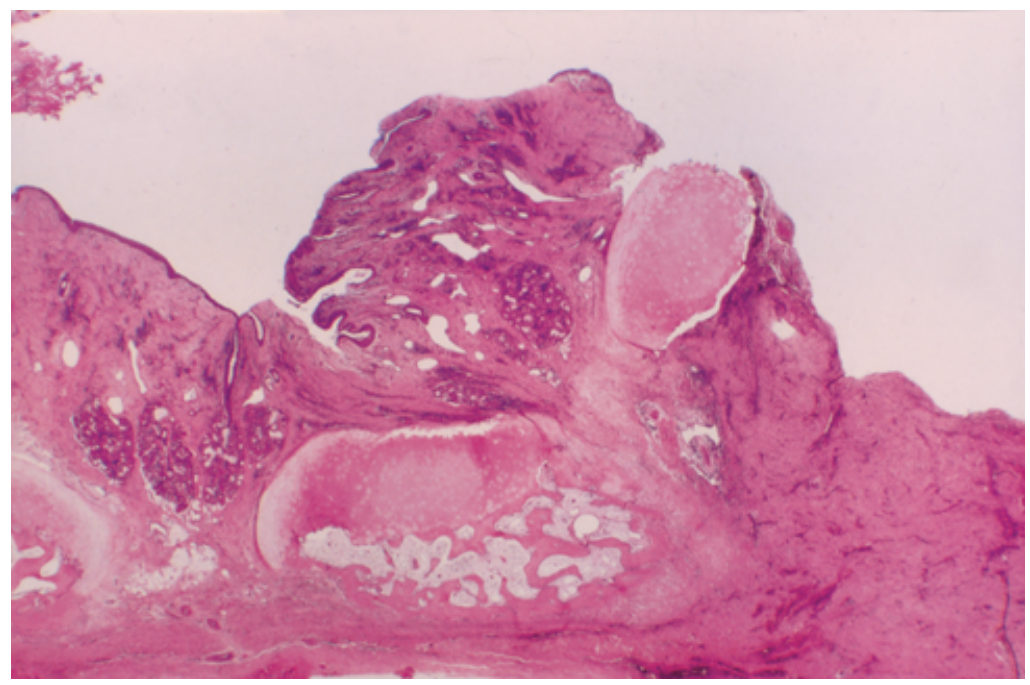

Figure 3 Photomicrograph of the surgical specimen showing squamous metaplasia of the tracheal mucosal epithelium and islands of cartilage with lamellar bone possessing haematopoietic marrow elements in the submucosa. Stain: haematoxylin and eosin; original magnification $\times 3.3$. choscopic examination after six weeks of treatment showed that the trachea had narrowed to a slit with the nodules increased in size. Spirometric testing showed a forced vital capacity of 1.591 ( $56 \%$ of predicted value) and forced expiratory volume in one second of 0.551 ( $20.6 \%$ of predicted value). The flowvolume loop revealed a pattern of fixed upper airway obstruction. Because of the persistent tracheal obstruction, and to determine the underlying tracheal disease, surgical resection was performed. The trachea was markedly adherent to the surrounding tissue. A $2 \times 1 \times 1 \mathrm{~cm}$ ill defined mass of tumour-like tissue was noted on the tracheal wall. The trachea was resected for $2.5 \mathrm{~cm}$ from the tracheostomy site to the cricoid cartilage. Suprahyoid release of the larynx and end-toend anastomosis of the trachea were performed. Pathological examination revealed squamous metaplasia of mucosal epithelium, islands of cartilage with foci of lamellar bone possessing haematopoietic marrow elements in the submucosa, and dense granulation tissue and fibrous tissue (fig 3). Gram staining showed no bacteria or sulphur granule-like material. Staining with Congo red was negative. The pathological diagnosis was tracheopathia osteochondroplastica. A review of the CT scan of the neck revealed calcified plaques and nodules along the lateral surfaces of the tracheal wall. After surgery the patient suffered from nosocomial pneumonia and respiratory failure and a tracheostomy was performed below the anastomotic site. After two months he was weaned off the ventilator. Follow up bronchoscopic examination showed granulation tissue (proved by biopsy) at the tracheostoma; however, the anastomotic site healed well and no residual tumour was found.

\section{Discussion}

Botryomycosis is an infection caused by nonfilamentous bacteria. Many pathogens have been identified as causative agents, including Staphylococcus aureus, Streptococcus spp, Peptostreptococcus spp, Proteus spp, Pseudomonas aeruginosa, Escherichia spp, Neisseria spp, Serratia marcescens, and Moraxella nonliquefaeiens. ${ }^{1-5}$ Peptostreptococcus spp is an organism of normal oral flora. Maves et al reported a case of Peptostreptococcus cultured from a botryomycotic lesion in a patient with a recurrent cheek mass. ${ }^{5}$ In the case presented here Peptostreptococcus spp was isolated from the pus and was compatible with the Gram-positive cocci identified by histological examination. Its low virulence is typical of isolates from botryomycosis lesions. ${ }^{45}$ Botryomycotic granules are composed of bacterial colonies that are surrounded by amorphous, eosinophilic, and radially arranged clubs (Splendore-Hoeppli material). ${ }^{1-5}$ They are indistinguishable from those of the actinomycosis and eumycetomas by haematoxylin and eosin staining and special stains are necessary for differential diagnosis. ${ }^{1-3}$ The presence of Grampositive cocci by Gram staining and the absence of branching hyphae by periodic acid-Schiff and Gomori's methenamine silver stains helped to establish the diagnosis of 
botryomycosis in this case. A fragment of cartilage accompanying the granules suggested an underlying cartilaginous disorder or metaplasia of chronic inflammation.

Neither the pathogenesis of botryomycosis nor the aetiology of tracheopathia osteochondroplastica has yet been determined. The following factors may be responsible for the pathogenesis of botryomycosis: (1) local trauma, (2) foreign body, (3) size of bacteria inoculum, (4) organisms of low virulence, and (5) immunodeficiency of host. ${ }^{2-5}$ Many aetiological factors have been proposed for tracheopathia osteochondroplastica including chronic infection, chemical or mechanical irritation, degeneration, metabolic factor, amyloidosis, ecchondrosis and exostosis, and metaplasia of elastic tissue. ${ }^{6-9}$

The concomitant occurrence of tracheal botryomycosis and tracheopathia osteochondroplastica is interesting. We propose that tracheopathia osteochondroplastica was the underlying disease and that the infection was established possibly by poor oral hygiene, aspirated peptostreptococci, ulcerated overlying mucosa, and the immunological defect of diabetes mellitus (which has been reported previously in a patient with botryomycosis ${ }^{2}$ ). Conversely, the osseous metaplasia of the cartilage and fibrous connective tissue could be a response to a longstanding chronic infection. ${ }^{9}$ Prolonged use of antibiotics for botryomycosis is usually required because the matrix covering the granules can be a barrier to the penetration of antibiotics. ${ }^{5}$ Thus, treatment with intravenous penicillin was continued for six weeks and the surgical specimen confirmed resolution

\section{Infection with feline Chlamydia psittaci}

\author{
Mark M Cotton, Martyn R Partridge
}

\begin{abstract}
A 48 year old man presented with malaise, fever, and progressive mental impairment. Chest radiography showed left lower lobe consolidation. A diagnosis of atypical pneumonia was made and, following treatment with erythromycin and acyclovir, his symptoms rapidly resolved. Asymptomatic biochemical hepatitis persisted for many weeks. Serological examination subsequently showed infection with feline Chlamydia psittaci.
\end{abstract}

(Thorax 1998;53:75-76)

\footnotetext{
Chest Clinic,

Whipps Cros

London E11 1NR, UK

M M Cotton

M R Partridge

Correspondence to:

Dr M R Partridge.

Received 20 September

1996

Returned to authors

4 November 1996

Revised version received

28 February 1997

Accepted for publication

1 April 1997
} referred as an emergency to the chest clinic by his general practitioner after a one week history of illness. On the first day of illness he felt generally unwell and had difficulty finding words. The next day he felt sick and vomited, and on of the infection. However, the inflammatory response to the infection led to granulation and fibrous tissue formation with further narrowing of the tracheal lumen, in spite of the fact that the bacteria were eradicated. ${ }^{10}$

This case is interesting for four reasons. Firstly, this is the first report of tracheal botryomycosis; secondly, the combination of tracheal botryomycosis and tracheopathia osteochondroplastica as a cause of airway obstruction suggests that the underlying disease should be sought in cases of tracheal botryomycosis; thirdly, the microbiological response of tracheal botryomycosis to laryngoscopic excision and antibiotic treatment was good; and fourthly, the interaction of the two diseases led to rapid progression of the tracheal stenosis.

1 Greenblatt M, Heredia R, Rubenstein L, Alpert S. Bacterial pseudomycosis ("botryomycosis"). Am f Clin Patho 1964; 41:188-93.

2 Omar T, Cooper K. Botryomycosis of the liver. Histopathology 1995;27:71-3.

3 Gillock CB, Sahni KS, Mumaw VR, Meier FA. Cerebra botryomycosis: case study. F Infect Dis 1990;162:765-7.

4 Katapadi K, Pujol F, Vuletin JC, Katapadi M, Pachter BR. Pulmonary botryomycosis in a patient with AIDS. Chest 1996;109:276-8.

5 Maves MD, Aguiar SW, Massanari RM, Robinson RA. Botryomycosis of the oral cavity. Head and Neck 1989;11:274-8.

6 Nienhuis DM, Prakash UBS, Edell ES. Tracheobronchopathia osteochondroplastica. Ann Otol Rhinol Laryngol 1990;99:689-94

7 Tukiainen $\mathrm{H}$, Torkko $\mathrm{M}$, Terho EO. Lung function in patients with tracheobronchopathia osteochondroplastica. Eur Respir f 1988;1:632-5.

8 Lundgren R, Stjernberg NL. Tracheopathia osteochondroplastica: a clinical bronchoscopic and spirometric study. Chest 1981;80:706-9.

9 Härmä RA, Suurkari S. Tracheopathia chondro-osteoplastica. A clinical study of thirty cases. Acta Otolaryngol 977;84:118-23.

10 Molloy AR, McMahon JN. Rapid progression of trachea stenosis associated with tracheopathia osteo-chondroplastica. Intensive Care Med 1988;15:60-2.

the fourth day was unsteady when walking. A diagnosis of food poisoning was made at a local casualty department and he was advised to rest. On days 5 and 6 he was febrile and delirious having visual hallucinations.

The patient had no past medical history of note. He had not travelled abroad and there was no history of bird contact although he had a healthy cat at home. There were no risk factors for HIV infection.

On examination he appeared well but tachypnoeic. Coarse crackles were heard in the left mid zone on auscultation of the chest. No focal neurological deficit was found, but assessment of higher mental functions was abnormal. Although orientated, he had sparse speech, poor concentration, difficulty in naming common objects, difficulty in reading, and could not write a sentence. He was completely unable to recall a simple address, and serial $7 \mathrm{~s}$ were only successful to 93 .

A chest radiograph revealed a left lower lobe pneumonia. A computed tomographic (CT) scan of the brain was normal. Full blood count including differential white cell count was normal, but his ESR was elevated at $70 \mathrm{~mm}$ in the first hour. No cold agglutinins were present. Apart from mild hyponatraemia $(\mathrm{Na} 130 \mathrm{mmol} /$ 1) the electrolytes were normal. He had a raised alkaline phosphatase of $227 \mathrm{IU}$ (normal < $120 \mathrm{IU}$ ) and alanine transaminase $145 \mathrm{IU}$ (normal $<40$ IU). Cerebrospinal fluid microscopy, protein 
Table 1 Serological results

\begin{tabular}{|c|c|c|c|c|c|c|}
\hline \multirow[t]{2}{*}{ Serovars } & \multicolumn{2}{|c|}{ Admission } & \multicolumn{2}{|l|}{ Day 6} & \multicolumn{2}{|c|}{ Day 16} \\
\hline & $\operatorname{Ig} G$ & $\operatorname{Ig} M$ & $\operatorname{Ig} G$ & $\operatorname{Ig} M$ & $\operatorname{Ig} G$ & $\operatorname{Ig} M$ \\
\hline Human $C$ psittaci & $1: 16$ & 0 & $1: 256$ & $1: 8$ & $1: 128$ & 0 \\
\hline Feline $C$ psittaci & $1: 16$ & 0 & $1: 256$ & $1: 128$ & $1: 128$ & 0 \\
\hline Guinea pig $C$ psittaci & $1: 16$ & 0 & $1: 64$ & $1: 8$ & $1: 64$ & 0 \\
\hline Avian $C$ psittaci & $1: 16$ & 0 & $1: 128$ & 0 & $1: 128$ & 0 \\
\hline IOL-207 human $C$ pneumoniae & $1: 16$ & 0 & 0 & 0 & 0 & 0 \\
\hline TW-183 human $C$ pneumoniae & $1: 16$ & 0 & 0 & 0 & 0 & 0 \\
\hline
\end{tabular}

and glucose were normal and blood cultures showed no growth. An EEG performed two days after admission was reported as compatible with mild encephalopathy but with no focal features to suggest herpes encephalitis.

He improved rapidly after five days of treatment with intravenous acyclovir $(10 \mathrm{mg} / \mathrm{kg})$ and two weeks of erythromycin ( $1 \mathrm{~g} /$ day; intravenously for five days), although at six weeks the alkaline phosphatase level remained raised (170 IU). Serological examination showed a rise in complement fixation titres for chlamydia group antibodies from less than 1:40 to 1:160, with no rise in titres for $\mathrm{Q}$ fever or mycoplasma. Specific serological tests from the chlamydial reference laboratory at the Institute of Ophthalmology in London demonstrated infection with feline Chlamydia psittaci (table 1) with a rise in specific IgM measured by microimmunofluorescence from undetectable to 1:128 (a $>1: 8$ titre usually taken as diagnostic). We were not able to test his cat serologically.

\section{Discussion}

Chlamydia psittaci is a pathogen for a wide range of avian and mammal species (including man) with a low host specificity. ${ }^{1}$ Eleven different serovars have been identified and one strain may produce more than one disease entity in a single species. ${ }^{2}$ Psittacosis is a well recognised infection in humans, classically transmitted from infected birds (ornithosis); person to person transmission is not thought to occur. A few cases of chlamydial respiratory tract infection in the UK have reported avian contact. $^{3}$

The diagnosis of chlamydial pneumonia is usually made on the basis of serological tests and the most commonly used method (complement fixation technique) detects only genus specific antibodies and cannot identify individual species. A fourfold rise in titres suggests recent infection. The microimmunofluorescence test, however, can detect IgM and IgG for particular species. An IgM titre of $>1: 8$ indicates a current or recent infection, although a convalescent sample should be taken for confirmation. ${ }^{34}$ Antigen detection techniques, either direct immunofluorescence or ELISA, have been used in the diagnosis of ocular and genital infections but not for sputum because of non-specific reactions. ${ }^{5}$ These techniques do not at present identify serovars. The polymerase chain reaction offers the opportunity to diagnose chlamydial infections ${ }^{6}$ and, as genetic analysis is matched to serology, should be able to identify serovars.

The most commonly reported mammalian source of chlamydial infection in humans are sheep where it is a cause of epidemic abortion. Abortion in women in contact with infected material has been caused by ovine $C$ psittaci. Meningitis, conjunctivitis, and pneumonia have also been reported. ${ }^{278}$

In cats, particularly kittens, Chlamydia commonly causes conjunctivitis. An antigenically distinct strain can cause a pneumonitis in cats. Cases of chlamydial conjunctivitis in cat owners have been attributed to feline transmission. $^{2}$ Excluding cases of conjunctivitis we could identify two previous reports of infection with feline $C$ psittaci. Griffiths et al reported a case of general malaise, cough, and abnormal liver function due to the feline serovar of $C$ psittaci in an immunosuppressed woman, ${ }^{9}$ and Regan et al reported a case of endocarditis with associated glomerulonephritis in a 40 year old man. ${ }^{10}$ Both cases reported close contact with cats. The subsequent death of the second case from pneumonia of an unknown aetiology raises the possibility of unrecognised immune deficiency in that case.

Cats are now the most popular pets in Britain. It seems likely that, since most cases of chlamydial infection are based on complement fixation tests and serovars of $C$ psittaci are not routinely identified, cases of feline chlamydial infection may be under recognised. It is unknown if extrapulmonary manifestations are more common with any particular serovar. We would recommend that serovars are identified in cases of atypical chlamydial infection and in those occurring in immunocompromised individuals. Since contact with cats is probably greater than with pet birds and chlamydial infection is widespread in the feline population, this may be the source of more infection than is presently recognised. However, changes in the provision of chlamydial reference laboratory services mean that species-specific serological testing is no longer routinely available.

\section{Addendum}

Follow up of this patient via his general practitioner has shown that he remains well four years later, suggesting that he does not have an underlying immunodeficiency.

1 Moulder JW, Hatch TP, Kuo C-C, Schachter J, Storz J. Chlamydia. In: Krieg NR, ed. Burgey's manual of systematic bacteriology. Volume 1. Baltimore: Williams and Wilkins, 1984: 729-39.

2 Johnson FWA. Chlamydiosis. Br Vet $\mathcal{f}$ 1992;139:93-101.

3 Wreghitt T. Chlamydial infection of the respiratory tract. CDR Review 1993;3:R119-24.

4 Treharne JD, Darouger S, Jones BR. Modification of the microimmunofluorescence test to provide a routine serodiagnostic test for chlamydial infection. $f$ Clin Pathol 1977;25:510-7.

5 Sillis M, White P, Caul EO, Paul ID, Treharne JD. The differentiation of Chlamydia species by antigen detection in sputum specimens from patients with communityacquired acute respiratory infections. $\mathcal{F}$ Infect 1992;25 (Suppl 1):77-86.

6 Tong CY, Sillis M. Detection of Chlamydia pneumoniae and Chlamydia psittaci in sputum samples by PCR. I Clin Pathol 1993;46:313-7.

7 Crosse BA, Gomes P, Muers MM. Ovine psittacosis and sarcoidosis in a pregnant woman. Thorax 1991;46:604-6.

8 Hadley KM, Carrington D, Frew CE, Gibson AA, Hislop WS. Ovine chlamydiosis in an abattoir worker. F Infect 1992;25(Suppl 1):105-9.

9 Griffiths PD, Lechler RI, Treharne JD. Unusual chlamydial infection in a human renal allograft recipient. $B M \mathcal{F}$ 1978;277:1264-5.

10 Regan RJ, Dathan JRE, Treharne JD. Infective endocarditis with glomerulonephritis associated with cat chlamydia (C psittaci) infection. $B r$ Heart $\mathscr{f}$ 1979;42:349-52. 